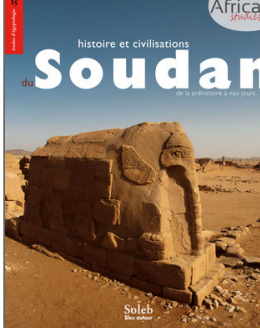

Histoire et civilisation du Soudan

De la préhistoire à nos jours

\title{
La condition féminine
}

\section{Odile Nicoloso et Nicolas Beaumé}

DOI : 10.4000/books.africae. 2972

Éditeur : Africae, Soleb, Bleu autour

Lieu d'édition : Paris, Khartoum

Année d'édition : 2017

Date de mise en ligne : 17 janvier 2022

Collection : Africae Studies

EAN électronique : 9782493207074

\section{OpenEdition}

\section{Books}

http://books.openedition.org

\section{Référence électronique}

NICOLOSO, Odile ; BEAUMÉ, Nicolas. La condition féminine In : Histoire et civilisation du Soudan : De la préhistoire à nos jours [en ligne]. Paris, Khartoum : Africae, 2017 (généré le 28 janvier 2022). Disponible sur Internet : <http://books.openedition.org/africae/2972>. ISBN : 9782493207074. DOI : https:// doi.org/10.4000/books.africae.2972. 


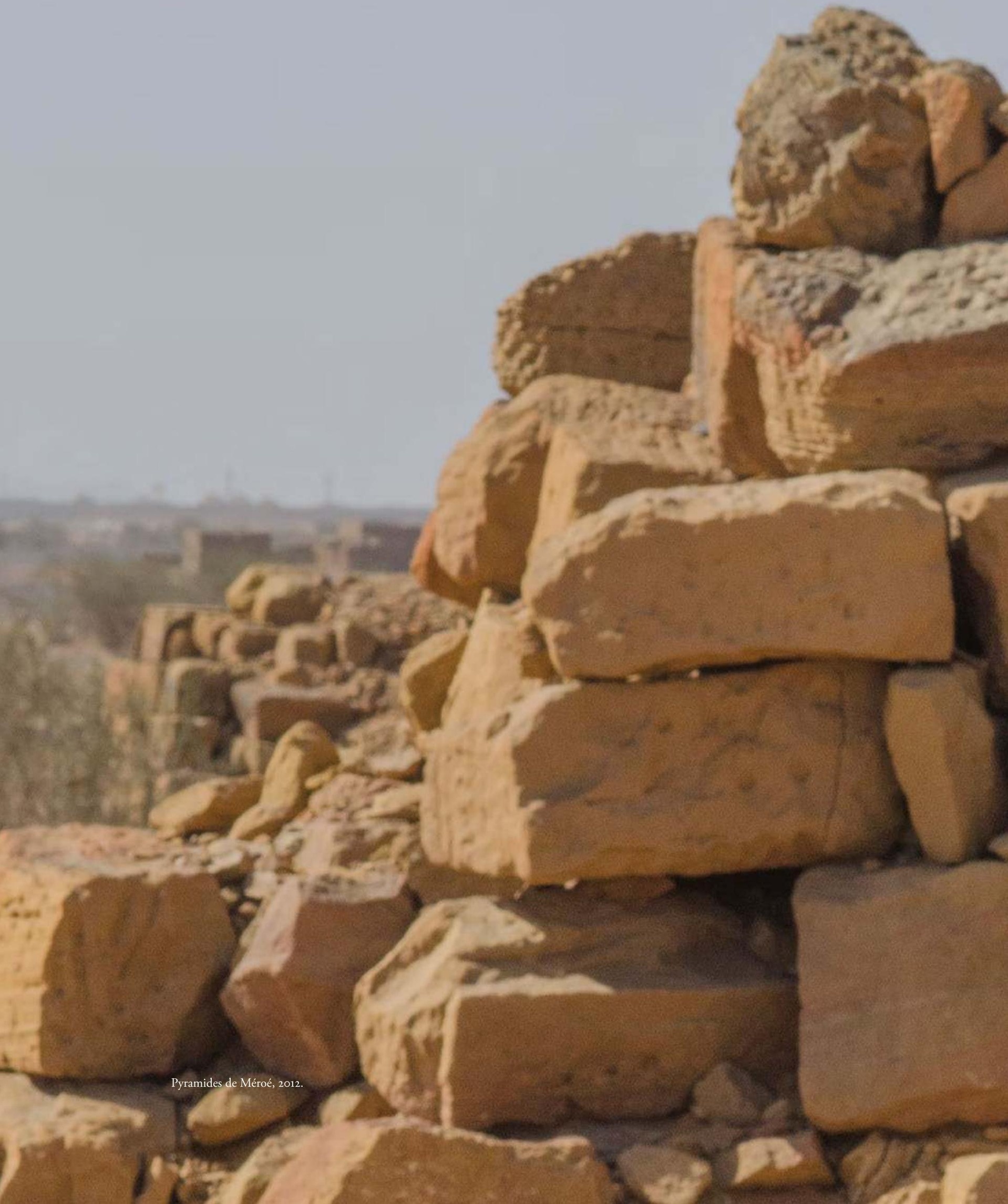




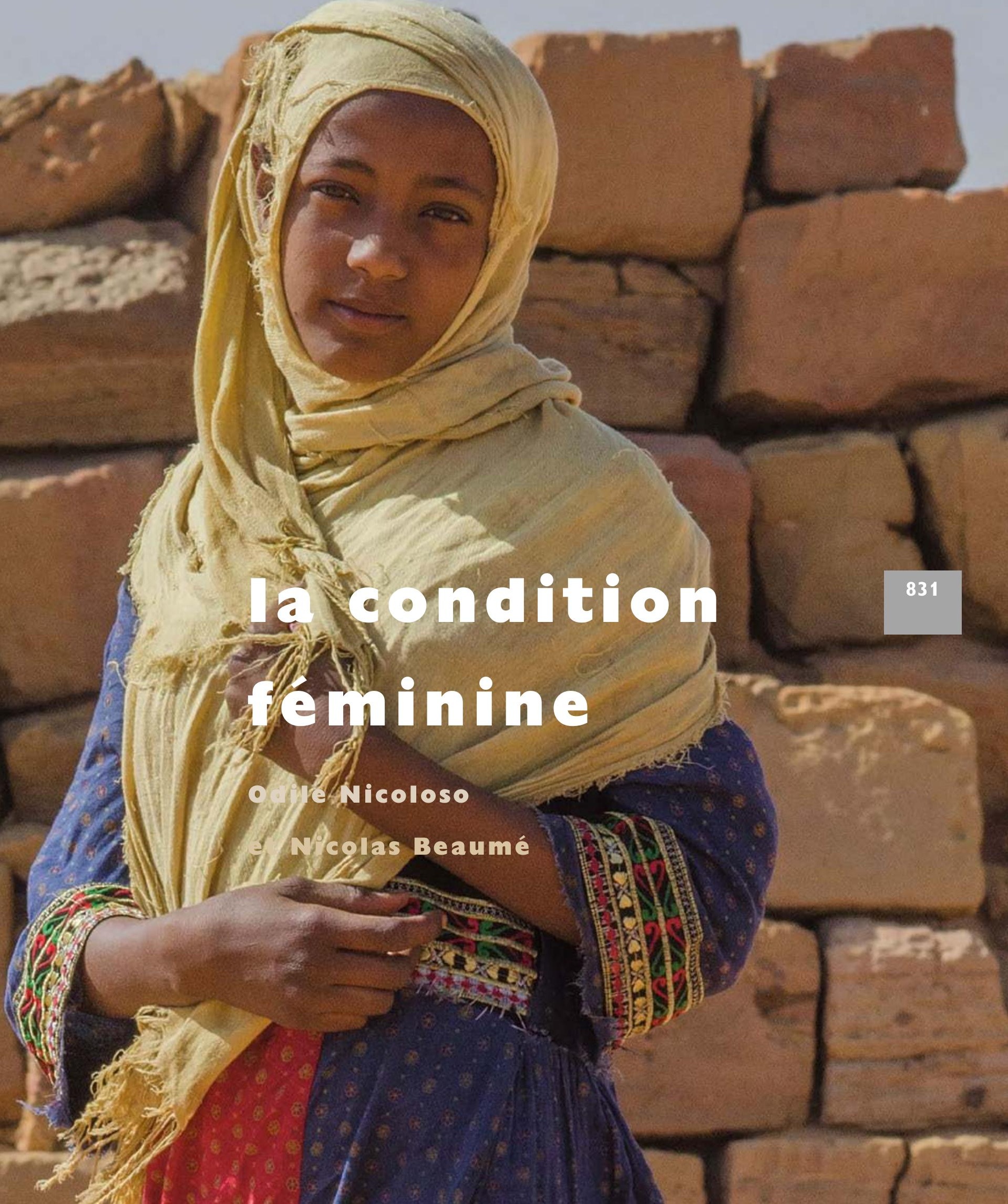




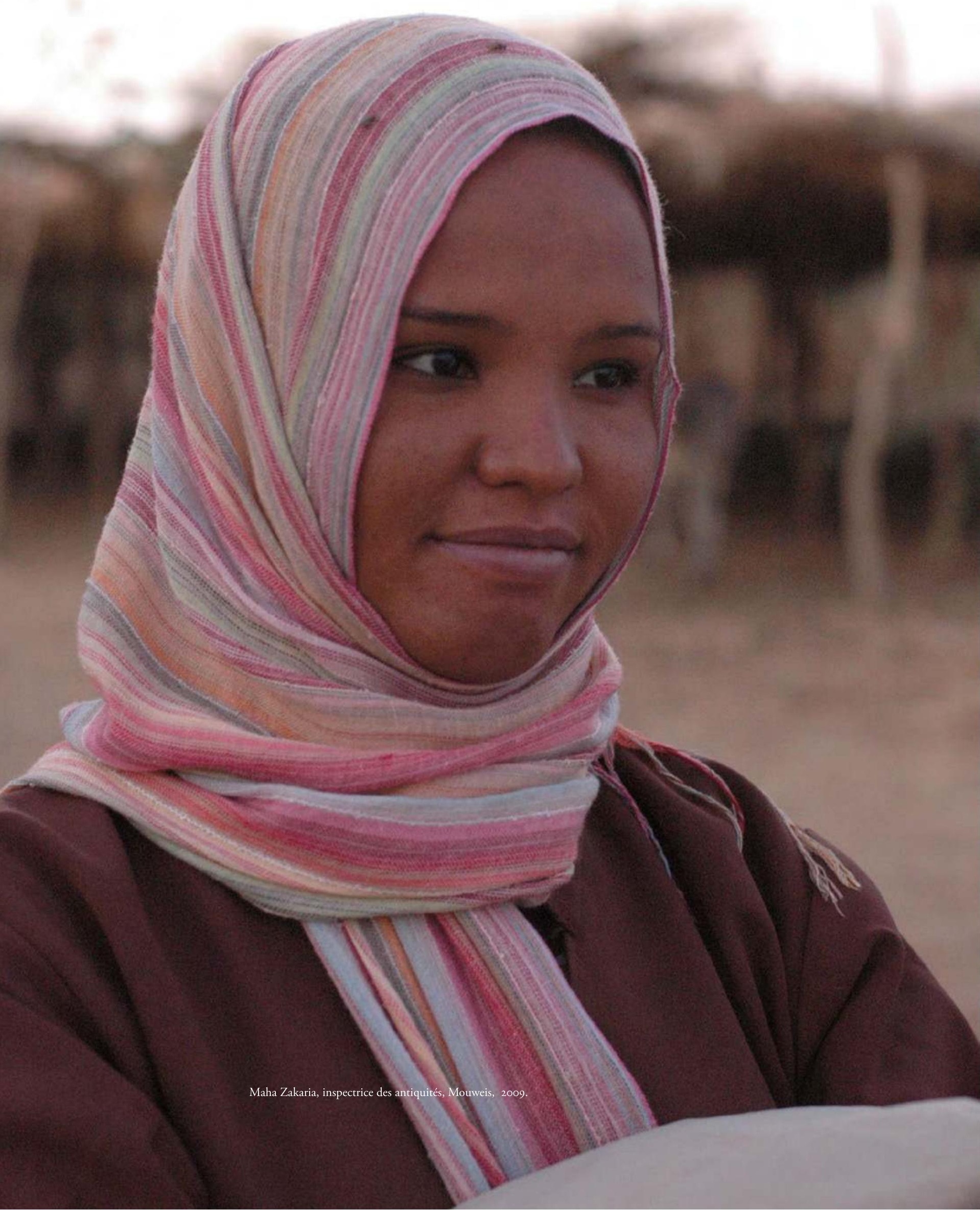


La situation des femmes est contrastée. Très présentes dans la vie publique du Soudan, elles sont nombreuses à exercer un métier et à travailler. La femme soudanaise est indépendante, active, elle n'est pas confinée dans la maison familiale et elle est libre de conduire et de vaquer librement à ses diverses occupations sans demander l'autorisation de son mari.

Les jeunes filles vont à l'université pour travailler ensuite. La première promotion d'étudiantes à terminer des études à l'université est sortie en 1930 (il existe actuellement 26 universités publiques et 5 grandes universités privées dont l'université Ahfad pour les femmes). La première association féminine a été fondée en 1924, la première femme médecin a été le docteur Khaleda Zahir nommée en 1949. Il y a actuellement 52\% de filles dans les universités (et $48 \%$ de garçons). Les familles payent fréquemment des études à l'étranger à ces derniers qui préféreront ensuite s'engager dans des voies concrètes comme le commerce ou l'immobilier, d'où l'expression «je vais dans le souk» pour signifier que l'on fait des études supérieures. Les femmes, elles, se dirigeront vers un travail plus régulier qui leur permettra d'équilibrer le budget familial, de s'occuper de leurs enfants et d'acquérir une indépendance financière. Bien sûr, le désir de s'instruire, de faire des études, est leur motivation première, mais souvent la situation économique actuelle, qui ne permet plus au chef de famille de subvenir seul aux besoins de sa famille est une incitation puissante. Au Darfour, par exemple, les femmes sont femmes d'affaires, commerçantes ou travaillent la terre... Les femmes ont obtenu le droit de vote en 1953 et participent à la vie politique (la Constitution leur réserve un quota de $25 \%$ de sièges à l'Assemblée nationale). Une femme dirige le parti socialiste soudanais et plusieurs sont ministres.

Malgré ce tableau plutôt positif, la femme est loin d'être l'égale de l'homme. Elle ne peut notamment se vêtir comme elle l'entend. La tenue traditionnelle de la femme est le tob, qui ressemble au sari indien. Son succès tient sans doute à sa simplicité — une seule pièce de tissu de 4,50 $\mathrm{m}$ de long sur $1,50 \mathrm{~m}$ - à son adaptabilité aux goûts de chacune — il est de couleurs variées - et à son efficacité: il protège des moustiques, du soleil... et des regards. Les élégantes le complètent par des chaussures et un sac à la mode. Les jeunes femmes ont adopté la jupe, voire le pantalon, mais normalement la peau est couverte ainsi que la chevelure. La grande majorité des femmes portent le hijab et certaines, sous l'influence, salafiste, le niqab (voile intégral). La police des mœurs veille au respect de la décence prescrite par la shari'a: aucune Soudanaise ne se risque à arborer un décolleté ou à porter une minijupe, et la journaliste Loubna a été arrêtée en 2009 pour avoir porté un pantalon.

Les femmes n'osent pas fumer la chicha ou même la cigarette en public. Rester en tête à tête avec un homme est malséant. On ne tolère pas qu'elles vivent seules, même quand elles sont veuves ou divorcées. La vie 

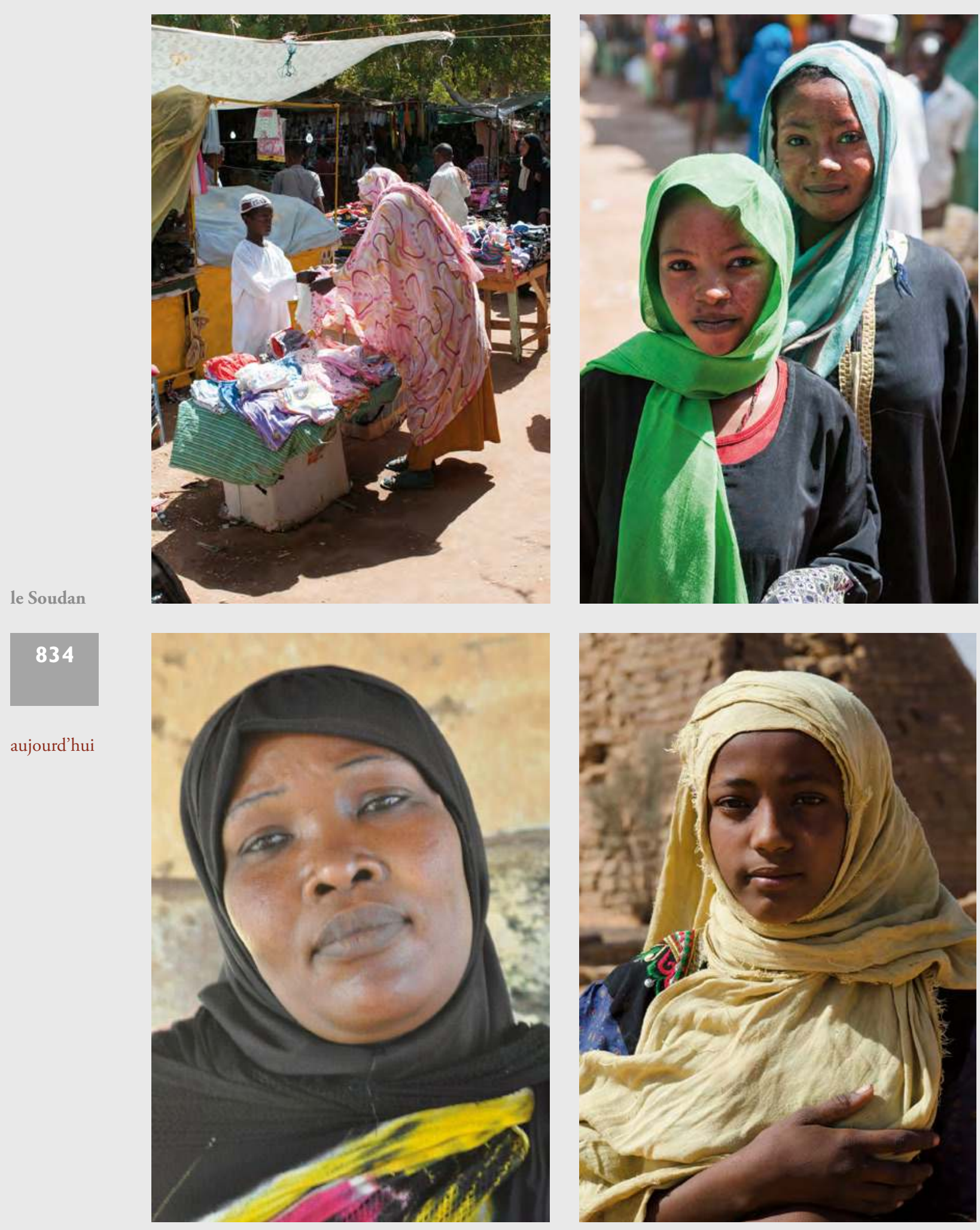
sociale des hommes et celle des femmes sont séparées; leurs conversations sont différentes; il ne peut être question de parler «sexe» ou de quelque autre sujet trivial devant la gent féminine; les repas ne sont pas mixtes, même en famille!

Dans le couple le mari détient tous les droits; la femme n'existe pas vis-à-vis de la loi; elle n'est pas imposable sur ses biens personnels. La contraception n'est autorisée qu'aux couples mariés car les relations sexuelles hors mariage sont interdites. Seule une veuve peut se remarier; dans ce cas, elle ne sera que deuxième ou troisième épouse.

La polygamie est autorisée au Soudan; l'homme peut prendre jusqu'à quatre épouses, à moins que celle qu'il a épousée en premier n'ait fait notifier l'exclusion de la polygamie dans le contrat de mariage. Le mari polygame doit accorder autant de temps à chacune de ses femmes (pour les nuits et les repas) et partager équitablement ses biens entre elles. Alors que les divorces sont de plus en plus répandus, la polygamie était un peu moins pratiquée, mais la montée des islamistes explique une recrudescence récente de cette pratique, qui, cela va sans dire, n'est pas particulièrement appréciée des Soudanaises.

La femme peut demander le divorce si son mari exerce sur elle des violences, s'il s'abstient de relations sexuelles ou s'il ne subvient pas à ses besoins matériels. Lors d'un divorce, les filles sont systématiquement confiées à la garde de leur mère jusqu'à leurs neuf ans et les garçons jusqu'à leurs sept ans. Les jugements tiennent de plus en plus compte de l'intérêt des enfants qui sont souvent confiés à leur mère jusqu’à un âge plus avancé. L'homosexualité, quant à elle, est un tabou absolu et est passible de la peine de mort.

\section{l'excision, une pratique qui perdure}

Khartoum, 2012;

Shendi, 2010;

Méroé, 2012.
Le Coran ne contient aucun texte faisant allusion de près ou de loin à l'excision des filles. Cette pratique «africaine», interdite par les Britanniques, n’a en fait jamais cessé et a repris après leur départ. Les petites filles la subissent à l'âge de six ou sept ans. Dénommée sunna, elle consiste en l'ablation du clitoris et des petites lèvres; les complications de cette mutilation traumatisante peuvent nécessiter des soins hospitaliers. Cette pratique, restée très répandue, épargne les chrétiennes. Dans plusieurs régions du pays, on pratique encore l'excision "pharaonique», cependant interdite par la loi soudanaise: en plus de l'excision "traditionnelle», la vulve est presque entièrement suturée; l'opération s'effectue souvent dans des conditions sanitaires si déficientes que nombre de petites filles y laissent leur vie! Les jeunes mères, heureusement, ont de plus en plus tendance à abandonner cette coutume. 
La circoncision n'est pas non plus, prescrite par le Coran. Elle n'est, elle aussi, mentionnée que par la sunna, d'authenticité controversée. C'est une tradition venue du monde arabe à laquelle adhèrent tous les Soudanais musulmans.

\section{soins de beauté et d'hygiène}

le Soudan

836

aujourd'hui

La pratique $\mathrm{du} d u k h a ̄ n$ (fumigation) est très répandue, surtout à partir du mariage. Un trou est creusé dans la cour ou le jardin : après y avoir mis des braises et du bois odorant, les femmes s'y assoient, le cou entouré d'une collerette qui retient la vapeur. C'est donc une sorte de sauna à l'issue duquel on met le dilka, substance exfoliante à base de plantes, puis du parfum extrait d'essences diverses et très odorantes comme le santal ou le musc: l'usage de certains parfums relève de véritables codes sensuels.

Après le dukhān, les femmes s'appliquent souvent le henné, qu'elles pratiquent depuis leur jeune âge (mais seules les femmes mariées s'en ornent les pieds). Le henné a été de tout temps le produit cosmétique le plus employé dans le monde arabe. Il occupe une place de choix dans les traditions musulmanes: fêtes de mariage, de circoncision, de l'Ä̈d... Le henné protège contre le mauvais œil, porte bonheur et, surtout, sublime la beauté. Aujourd'hui il est utilisé pour le plaisir de se teindre les cheveux ou d'avoir un beau motif tatoué temporairement sur le corps. Il est aussi considéré comme une plante médicinale de par ses différents composants dont le tanin. Le henné est cultivé intensivement au Soudan. Les hommes l'utilisent aussi parfois pour se teindre barbe ou cheveux blancs. L'épilation est très répandue.

\section{les tea ladies, ou dames du thé}

À presque tous les coins de rue de Khartoum, des femmes proposent du thé au gingembre ou à la cardamome, des cafés très sucrés ou épicés, du karkadé, du tabaldi, parfois des beignets. Ce sont les sittat chay (settāt eš-šay, singulier sitta chay, sett eš-šay). Elles sont des centaines. Leur réputation dépend de l'emplacement de leur commerce et de leur propreté. Aux endroits les plus animés de la capitale, elles installent sur les trottoirs ombragés, au ras du sol, petites tables et tabourets. Ce sont pour la plupart des femmes du peuple,

Double page

suivante:

Souhaïba, Moua'da, Moawwiya et Mona, Saï 2015. pauvres et analphabètes, des veuves ou des réfugiées qui viennent du Sud ou d'Éthiopie. Il existe cependant des cas atypiques, comme une universitaire devenue veuve ou des hommes (Béja, pour la plupart) dans le besoin, qui font ainsi vivre une nombreuse famille. Comme leur activité n'est pas soumise à l'octroi d'une licence et est donc seulement tolérée, il arrive que la police les chasse et que leur matériel soit confisqué 

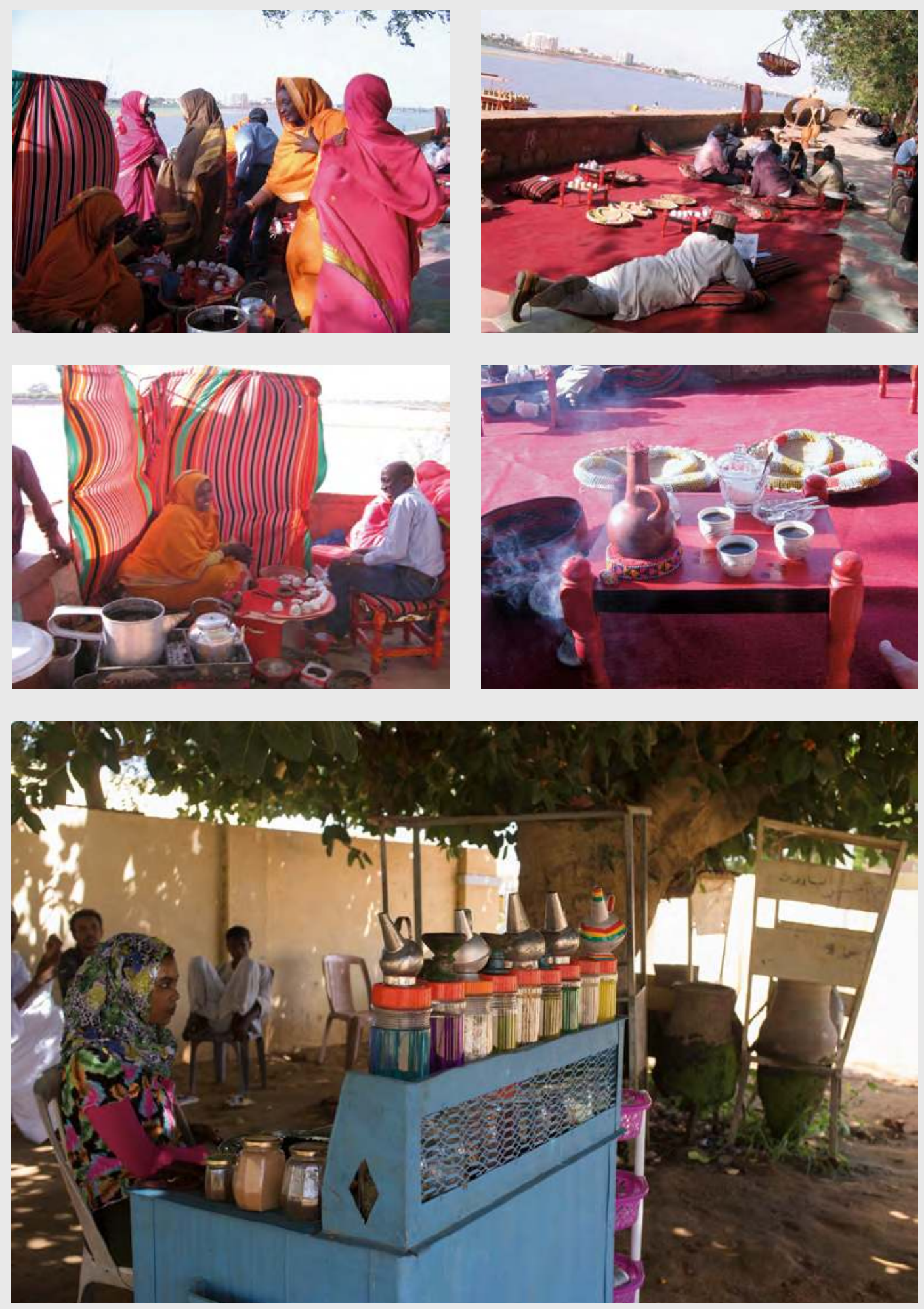

Sittat chay: à Khartoum, en 2011 (en haut), et à Shendi, en 2013 (en bas). 


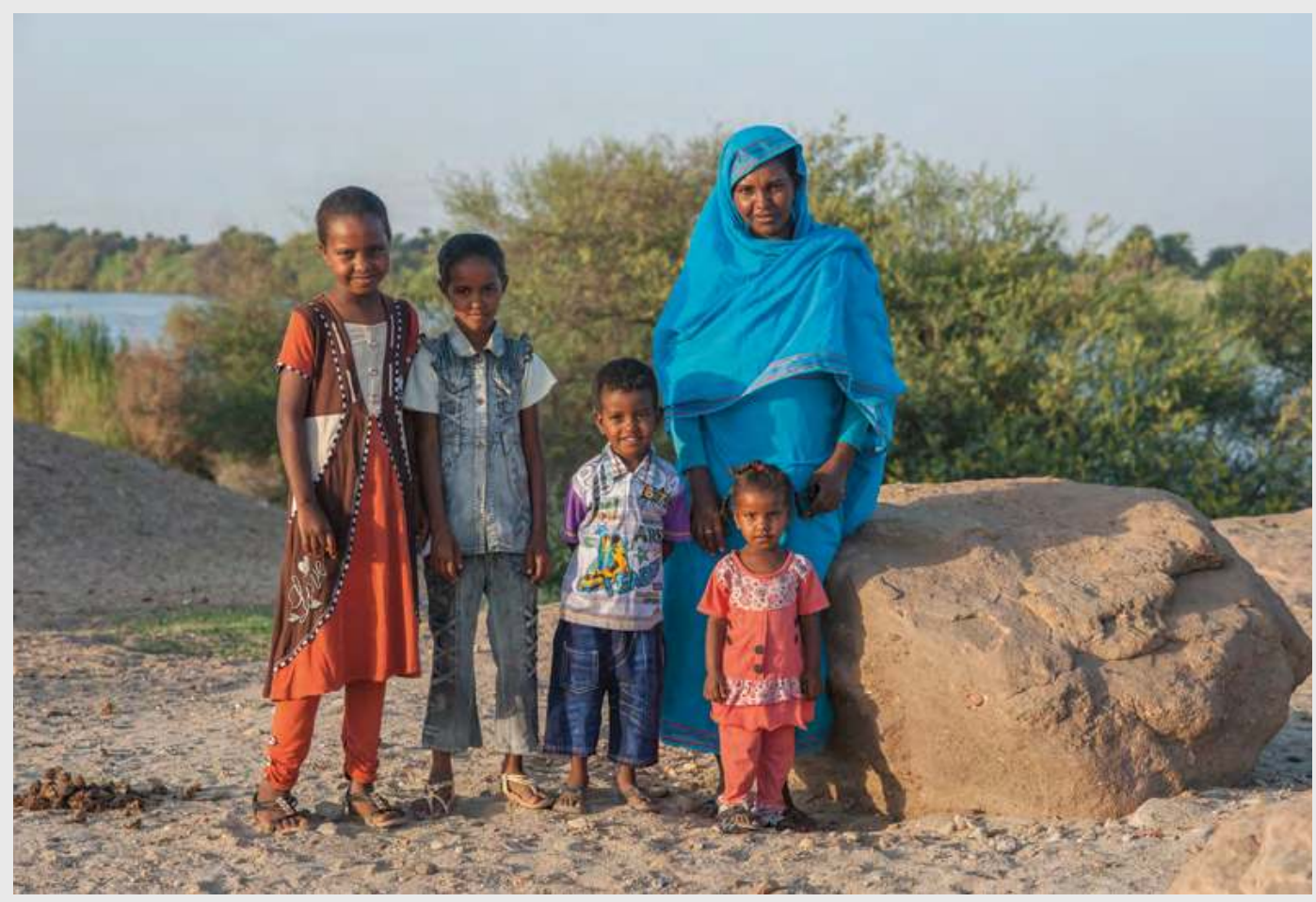

le Soudan

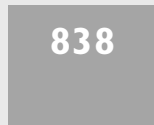

aujourd'hui
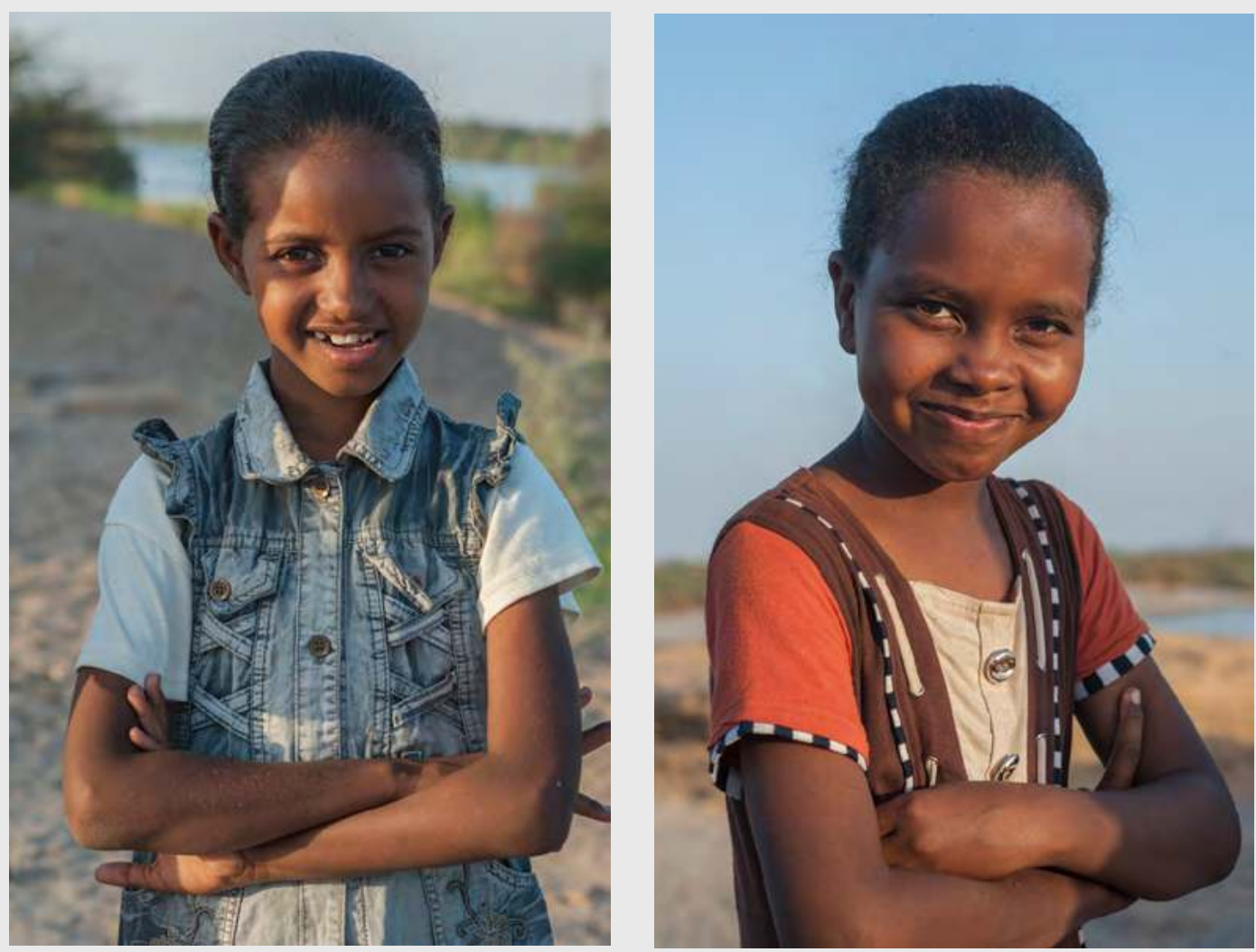
